\title{
DESDE LA TIERRA DE NUNCA JAMAS: México y la epidemia de Covid-19
}

\section{FROM THE EARTH OF NEVER JAMAS: Mexico and the Covid-19 epidemic}

DA TERRA DO NUNCA JAMAIS: México e a epidemia covid-19

\author{
Regina Martínez Casas \\ Centro de Investigaciones y Estudios Superiores en Antropología Social (CIESAS) \\ reginamc@ciesas.edu.mx \\ Ciudad de México \\ Elena Hernández Maldonado \\ elenazmaldonado@gmail.com
}

\section{Resumen}

La redacción de este artículo la realizamos después de que llevamos más de 250 días encerrados en nuestras casas a raíz de que se nos pidió a todos los mexicanos que, si podíamos hacerlo, nos quedáramos en casa. Formamos parte del cada día mas reducido grupo de personas que puede -o quiere- mantener el confinamiento a pesar de lo que representa trabajar fuera de la oficina, no poder reunirnos en persona con nuestros colegas, amigos y familia y depender de la información que podemos gestionar de manera remonta. En el presente artículo se presentan algunos de los efectos sociales del confinamiento y la crisis sanitaria en México. Más que contabilizar contagios y fallecimientos, nos interesa destacar las consecuencias de la contingencia en la salud mental, la violencia y la deserción escolar en hogares mexicanos, especialmente entre las mujeres. También incluiremos algunas historias de familias afectadas por la pandemia y recuperaremos entrevistas realizadas en torno a la experiencia de la enfermedad en la vida de las personas.

Palabras clave: pandemia, crisis sanitaria, confinamiento, efectos sociales, Mexico. 


\begin{abstract}
The writing of this article was done after we had been locked in our homes for more than 250 days because all Mexicans were asked to stay at home if we could do so. We are part of the smallest group of people who can - or want - maintain confinement despite what it means to work outside the office, not be able to meet in person with our colleagues, friends and family and depend on the information we can manage in a way that goes back. This article presents some of the social effects of confinement and the health crisis in Mexico. Without accounting for contagion and deaths, we are interested in highlighting the consequences of contingency on mental health, violence and dropout in Mexican households, especially among women. We will also include some stories of pandemic-affected families and retrieve interviews about the experience of the disease in people's lives.
\end{abstract}

Keywords: pandemic, health crisis, confinement, social effects, Mexico

\title{
Resumo
}

A redação deste artigo foi feita depois de ficarmos trancados em nossas casas por mais de 250 dias porque todos os mexicanos foram convidados a ficar em casa se pudéssemos fazê-lo. Fazemos parte do menor grupo de pessoas que podem - ou querem - manter o confinamento apesar do que significa trabalhar fora do escritório, não poder nos encontrar pessoalmente com nossos colegas, amigos e familiares e depender das informações que podemos gerenciar de uma forma que remonta. Este artigo apresenta alguns dos efeitos sociais do confinamento e da crise de saúde no México. Sem contabilizar contágio e mortes, estamos interessados em destacar as consequências do contingenciamento em saúde mental, violência e abandono nos domicílios mexicanos, especialmente entre as mulheres. Também incluiremos algumas histórias de famílias afetadas pela pandemia e resgataremos entrevistas sobre a experiência da doença na vida das pessoas.

Palavras-chave: pandemia, crise de saúde, confinamento, efeitos sociais, México 
a redacción de este artículo la realizamos después de que llevamos más de 250 días encerrados en nuestras casas a raíz de que se nos pidió a todos los mexicanos que, si podíamos hacerlo, nos quedáramos en casa. Formamos parte del cada día más reducido grupo de personas que puede $-\mathrm{o}$ quieremantener el confinamiento a pesar de lo que representa trabajar fuera de la oficina, no poder reunirnos en persona con nuestros colegas, amigos y familia y depender de la información que podemos gestionar de manera remonta. En el presente artículo se presentan algunos de los efectos sociales del confinamiento y la crisis sanitaria en México. Más que contabilizar contagios y fallecimientos, nos interesa destacar las consecuencias de la contingencia en la salud mental, la violencia y la deserción escolar en hogares mexicanos, especialmente entre las mujeres. También incluiremos algunas historias de familias afectadas por la pandemia y recuperaremos entrevistas realizadas en torno a la experiencia de la enfermedad en la vida de las personas.

En marzo de 2020 México reportó los primeros casos confirmados de Covid-19 y el primer fallecimiento. Desde finales de enero se había empezado a informar a la población que era inevitable que el virus llegara a estas tierras, pero que había ya una estrategia trazada por el gobierno para atender la contingencia de salud. Quedaba claro que esta estrategia incluía una propuesta de comunicación encabezada por el Subsecretario de Salud Hugo López Gatell, quien todos los días, a las 19:00 horas, se presenta en una conferencia de prensa en la que se informa el estado diario de la contingencia. Se incluye información nacional sobre contagios, casos activos $\mathrm{y}$ fallecimientos, pero también se incluye un panorama mundial $\mathrm{y}$, en ocasiones, se desagrega la información por estados o se habla la disponibilidad hospitalaria. Este último tema ha cobrado creciente importancia en las conferencias vespertinas porque es uno de los más importantes criterios para determinar el control de la pandemia.

Es notable que el formato de estas comunicaciones no haya cambiado en más de 250 días, pero los contenidos y la manera de presentar la información sí ha evolucionado con el tiempo. En marzo se estimaba que México tendría pocos contagios y muertes, en ese momento el foco en China estaba bajo control y Europa estaba muy activa en contagios $y$ fallecimientos. A mediados de ese mes se invitó a la población mexicana a quedarse en casa y se cancelaron actividades en escuelas, oficinas, fábricas y servicios no esenciales. Parte de los mensajes que se han compartido de manera cotidiana en estas conferencias vespertinas han incluido 
valoraciones sobre los perfiles de quienes se contagian y enferman gravemente e incluso fallecen. Desde el inicio se ha tejido una retórica sobre las llamadas comorbilidades: el sobre peso, la diabetes y la edad, pero también se generó un conjunto de valoraciones de tipo social. El virus llegó desde fuera por culpa de los viajeros ricos que lo importaron de Europa o de Estados Unidos. Las personas corruptas se enferman y fallecen más que las honradas. El clima y la raza mexicana impedirían la propagación de la enfermedad. Por otro lado, la reticencia de algunos funcionarios públicos de alto nivel de usar cubrebocas en público ha generado una fuerte polémica. Un fenómeno similar se ha registrado en Estados Unidos y en Brasil. En los tres países, el uso $-\mathrm{o}$ no- de esta medida de protección se ha convertido en una posición política, pero también una reproducción de las relaciones de poder.

La nuestra no es una reflexión médica ni busca cuestionar los argumentos sobre los factores que agravan a muchos de los enfermos de Covid-19. Sin embargo, buscamos mostrar que parte de la política diseñada para atender la pandemia ha estado fuertemente cargada de prejuicios $y$ contradicciones. Por otro lado, nos centraremos en los efectos sociales que ha tenido el confinamiento y sus potenciales secuelas a mediano plazo. En especial, nos interesa destacar que algunas de las contradicciones han tenido consecuencias en las estrategias gubernamentales para atender la pandemia y que han generado respuestas en la población que probablemente han agudizado su gravedad. Al finalizar septiembre del 2020 México es uno de los países con más casos de contagios y una de las tasas de fallecimientos más altas del mundo. Más del $10 \%$ de los casos diagnosticado como positivos fallecen. Por otro lado, la cancelación parcial de actividades ha tenido graves consecuencias en la economía, en la organización de las familias y en el sistema educativo.

\section{La narrativa de la pandemia}

A menos de dos meses de la primera defunción, el gobierno de México empezó a insistir en que la epidemia estaba bajo control, aunque se había advertido en el mes de febrero que sería un proceso largo. En el mes de marzo se inició la denominada jornada de sana distancia que invitaba a confinarse de manera más o menos voluntaria. Desde la fase inicial de las estrategias de comunicación para la población mexicana llamó la atención la constante tensión entre las declaraciones del responsable de organizar la gestión de la pandemia-el subsecretario de la Secretaría de Salud- y las declaraciones del presidente de México: 
Lunes 27 de abril 2020 (Andrés Manuel López Obrador)

Vamos bien, entonces si no nos relajamos en disciplina ${ }^{I}$, es decir, si cumplimos las medidas vamos a tener buenos resultados (...) si nuestro país queda mal visto en el extranjero porque, toco madera, se nos agravara la situación sanitaria entonces eso nos afecta nada más piensen por ejemplo en el turismo: si sale de control la epidemia en México en las zonas turísticas, cuando regresemos a la normalidad en el mundo se va a pedir información de donde o a donde ir entonces es un esfuerzo pero no es en vano y lo mas importante desde luego es que no se pierdan vidas humanas, hasta en términos económicos, nos conviene que se respeten las medidas porque se están obteniendo buenos resultados y si seguimos así vamos a levantar la cuarentena y vamos a reactivar la economía u el daño económico va a ser menor y con lo que estamos haciendo de garantizar el bienestar de la gente, pues se va a lograr el renacimiento en nuestro país pronto va a ser una crisis transitoria que además, nosotros seguimos los parámetros, recomendaciones recetas del neoliberalismo y por eso llevamos ventaja, imagínense si no vamos a llevar ventaja ${ }^{2}$

Para esa fecha había un poco más de 1000 muertes por Covid-19 confirmadas en México y nuestras cifras se veían muy distantes de los números que, en ese momento, se registraban en otros países de la región y del mundo. Incluso ha sido frecuente comparar las cifras de México con

\footnotetext{
${ }^{1}$ Las negritas son nuestras

${ }^{2} \mathrm{https} / / / \mathrm{www}$.youtube.com/watch?v=Ub2uEXFv1lgç.
}

las de países europeos y del continente americano como parte de la retórica para mostrar el buen manejo de la pandemia durante las conferencias de prensa diarias.

\section{Martes 28 de abril 2020 (Andrés Manuel López Obrador)}

Todavía viene un tiempo difícil, afortunadamente hemos podido aplanar la curva de los infectados de los casos de coronavirus, sucedió lo que nos temíamos y nos preocupaba que se saliera de control la pandemia y que no se tuvieran las camas, los ventiladores, los médicos para atender a los enfermos entonces se evitó por eso con las medidas que se tomaron, lo que está haciendo la gente que no voy a dejar de reconocerlo, de agradecerlo ${ }^{3}$

Justo en el mes de abril se anunciaron compras de emergencia en el extranjero de equipo médico y de protección personal. La crisis sanitaria estaba en el peor momento de la primera oleada en Europa y Estados Unidos y el acceso a estos equipos era difícil y muy costoso. El gobierno de México determinó que, con motivo de la emergencia, dejarían de atenderse los mecanismos gubernamentales que aseguran la transparencia y eficiencia en los procesos de compras a gran escala y se le encomendó a canciller (el Secretario de Relaciones Exteriores) encabezar las

\footnotetext{
3 https://aristeguinoticias.com/2904/mexico/amlo-dijoque-se-esta-aplanando-la-curva-pero-en-la-fase-3-loscontagios-son-exponenciales-video/
} 
negociaciones con empresas médicas y farmacéuticas a nivel mundial para adquirir los equipos y medicamentos. La falta de transparencia en los procesos de compra de insumos médicos y en su distribución ha generado cuestionamientos $\mathrm{y}$, como veremos más adelante, graves consecuencias en las condiciones de riesgo en las que labora el personal de salud en México.

\section{Lunes 4 de mayo 2020 (Hugo López Gatell)}

En la última semana hemos compartido esta noticia positiva de que no solo por observación directa como lo hemos venido documentando todos los días, sino también por las proyecciones matemáticas ya están en la parte de declive: Cancún por ejemplo, que hace unos días anunciamos que estaba en el punto medio, en este momento parece estar ya en una tendencia hacia abajo. Ahora, muy importante, esto no quiere decir que ya se pueden suspender las medidas de sana distancia: si lo hiciéramos en este momento, tendríamos el grave peligro de que reemerja la epidemia en la medida en que todavía existen personas infectadas, personas con enfermedad, estas pueden transmitir a otras personas. ${ }^{4}$

La fluctuación en la tasa de contagios y fallecimientos ha sido notable desde el mes de mayo y hasta la fecha de escritura de este

\footnotetext{
4 https://www.gob.mx/presidencia/es/articulos/versionestenografica-conferencia-de-prensa-informe-diariosobre-coronavirus-covid-19-en-mexico241579? idiom=es
}

texto (octubre de 2020). Sin embargo, quien suele llamar la atención sobre los riesgos de rebrotes es el subsecretario de salud. El resto de las autoridades federales suelen alinearse a los llamados de la presidencia de la república para retomar actividades que permitan activar una economía fuertemente golpeada por la crisis, no así los gobiernos locales que son quienes enfrentan muchas de las consecuencias cotidianas de la pandemia. Cuando en Europa se habían ya controlado los contagios y los fallecimientos, pero se hablaba de los riesgos de una segunda ola de contagios, el discurso dominante en México era de éxito en los resultados de la gestión epidémica. Después de la llamada jornada nacional de Sana Distancia iniciada en marzo de 2020, se estableció un semáforo epidemiológico para retomar de manera gradual actividades productivas, no así las escolares a partir del verano.

\section{Martes 9 de junio 2020 (Andrés Manuel López Obrador)}

México está dando un ejemplo en el mundo porque logramos aplanar esta curva y evitar que se saturaran los hospitales sin medidas coercitivas, sin el uso de la fuerza. Con la participación voluntaria, consciente de los ciudadanos. Esto no se logró en otras partes del mundo 5 ejemplo-al-mundo-en-control-de-epidemia-de-covid19-presidente-manejo-ha-sido-eficaz-y-con-apoyo-delpueblo-afirma/ 


\section{Martes 6 de julio Andrés Manuel López Obrador)}

Aquí aprovecho para agradecerle al pueblo de México por el sacrificio, por el buen comportamiento sin necesidad de medidas autoritarias la gente ha actuado en forma responsable y esto nos ha permitido enfrentar la pandemia que no falten las camas, que no falte equipo, que no falten especialistas, que no falten médicos, que se atienda a la población porque si se logró aplanar la curva, no se ha desbordado el sistema médico , esto ha significado también salvar vidas, entonces no ha sido en vano el sacrificio de la gente ${ }^{6}$.

\section{Martes 14 de julio 2020 (Andrés Manuel López Obrador)}

La estrategia seguida con el fin de atender la pandemia de coronavirus fue clave para aplanar la curva, lo que permitió levantar al sistema de salud, que estaba en el suelo, y contar con las camas, los ventiladores y tener los médicos ${ }^{7}$

Con relación a estas intervenciones del presidente, una semanas después, la Organización Aministía Internacional mostró que México es el país en donde la tasa de mortalidad entre el personal de salud es la más alta del mundo y cuestiona los mecanismos que se han utilizado para proteger al personal que trabaja en hospitales, no solo a los

\footnotetext{
6 https://www.reporteindigo.com/reporte/amlo-se-logroaplanar-la-curva-de-contagios-no-ha-sido-en-vano-elsacrificio-de-la-gente/

https://www.jornada.com.mx/ultimas/politica/2020/07/ 15/estrategia-del-gobierno-clave-para-aplanar-la-curvaamlo-1284.html
}

médicos, sino al personal de enfermería, laboratorios, limpieza y vigilancia.

El estudio referido señala que el país había reportado hasta ese momento $\mathbf{1 , 3 2 0}$ decesos confirmados por COVID-19 entre personal de salud, por encima de los mil 77 de Estados Unidos. Steve Cockburn, director de justicia económica y social en Amnistía Internacional, consideró que el hecho de que más de7 mil trabajadores de salud hayan muerto en el mundo representa "una crisis de dimensiones alarmantes". Para principios de septiembre de 202097 mil 632 enfermeros, doctores y otros empleados de hospitales en México han sido diagnosticados con coronavirus desde que comenzó la pandemia (42\% enfermeros, $31 \%$ técnicos, asistentes y personal de limpieza y mantenimiento y $27 \%$ médicos $)^{8}$

\section{Viernes 7 de agosto 2020 (Andrés Manuel López Obrador)}

Va a pasar esta pandemia, tiene que ceder, ya está sucediendo, aun con lentitud ya está bajando, quisiéramos que avanzáramos más, que desapareciera la pandemia, para que no se causaran sufrimientos, pero bueno, tenemos que enfrentar esta pandemia y lo estamos haciendo ${ }^{9}$

\footnotetext{
${ }^{8}$ https://www.elfinanciero.com.mx/salud/mexico-primerlugar-mundial-en-personal-de-salud-fallecido-porcovid-19-amnistia-internacional

9 https://www.forbes.com.mx/politica-amlo-estrategiacovid-19-aplano-curva/
} 
Esta intervención coincidió con las fechas en las que las cifras oficiales registraron cifras récord de contagios y fallecimientos y la Ciudad de México amenazaba con medidas de reconfinamiento de la población, pero en más de la mitad del país se declaraba una apertura de espacios públicos. Sin embargo, las actividades educativas continúan ofreciendóse a distancia y se diseñó un modelo híbrido con clases por televisión e interacciones vía electrónica entre docentes y estudiantes que ha sido fuertemente cuestionado. Cifras de la propia Secretaría de Educación Pública plantean que más del $15 \%$ de la población estudiantil no se reinscribió para el periodo de otoño de 2020. Esto afectó particularmente a los niños en preescolar y a los jóvenes en educación superior.

A finales de agosto la pandemia dejó de ser un tema recurrente en las conferencias de prensa que todas las mañanas se trasmiten desde Palacio Nacional, sede el gobierno federal y residencia del presidente de la república. En esa coyuntura, algo que llamó la atención a la población mexicana es que el mensaje de las fiestas patrias que en México se celebra los días 15 y 16 de septiembre, no se haya hecho mención a las personas fallecidas por Covid-19. Esta conmemoración sin embargo sí sucedió alrededor de la fiesta de muertos que en México tiene una gran relevancia social y cultural. El presidente decretó tres días de luto nacional por las víctimas de la Covid que coincidían con las fechas de dicha celebración (31 de octubre, 1 y 2 de noviembre).

\section{La crisis sanitaria $y$ económica para las mujeres en México.}

La pandemia ha afectado a toda la población de México y del mundo, pero no a todos los ha afectado de la misma manera. En México ha afectado de manera mucho más evidente a las mujeres. Unos días antes de que se declarara la jornada nacional de sana distancia, en marzo de 2020 centenares de miles de mujeres salimos a las calles a exigir equidad y el fin de la violencia machista. El domingo 8 de marzo las calles de la Ciudad de México y de las principales ciudades del país se tiñeron de morado bajo la consigna de "ni una más". El lunes 9 de marzo una huelga de mujeres dejó desiertas las calles del país. Fue casi un augurio de lo que sucedería unos días después cuando se invitó al confinamiento, pero la huelga de mujeres y la marcha fueron duramente descalificadas por el presidente de México que acusó de oscuras motivaciones neoliberales a quienes la convocaron.

El hecho es que en México, el incremento en las denuncias a los teléfonos de emergencia por violencia doméstica se incrementaron $45 \%$ en el segundo y tercer 
trimestre del 2020 (abril a junio y julio a septiembre) y en una encuesta telefónica realizada por el Instituto Nacional de Estadística y Geografía (INEGI) en mayo de $202018 \%$ de las mujeres reportaron haber sufrido episodios de violencia dentro de sus hogares durante el confinamiento. Las carpetas de investigación por feminicidios se incrementaron $40 \%$ entre mayo y junio. De abril a septiembre se han registrado 473 víctimas de feminicidio y mil 443 mujeres han sido víctimas de homicidio doloso, esto significa que cada día 10 mujeres son asesinadas en México. Aunado a ello, las denuncias por violación aumentaron 22 por ciento de abril a junio y la trata de personas 13.4 por ciento $^{10}$.

Adicionalmente se ha registrado una tasa de desempleo más alta entre mujeres. $64 \%$ del total de las personas que perdieron sus empleos desde marzo (12.5 millones de personas) son mujeres y la mayoría no los ha recuperado según reporta el propio INEGI y el Instituto Mexicano de Seguro Social ${ }^{11}$. Una de las explicaciones que se plantean es que los cuidados de niñas, niños, adolescentes y adultos mayores recae en las mujeres y muchas de ellas han optado por dejar de buscar empleo mientras no se reanuden las actividades escolares presenciales y los

\footnotetext{
10

https://www.milenio.com/politica/comunidad/coronavir us-alertan-crisis-expone-violencia-mujeres

${ }^{11}$ https://www.elfinaciero.com
}

adultos mayores estén en menos riego que el que presentan actualmente.

La literatura feminista (Esteban 2017; Rodríguez 2015) ha documentado desde hace más de dos décadas la importancia del papel de las mujeres como cuidadoras en el ámbito de las familias en América Latina. A pesar de que el confinamiento replanteó la división del trabajo doméstico, en la mayoría de los hogares mexicanos la responsabilidad del cuidado sigue siendo un tema femenino. Por otro lado, el incremento en el tiempo que pasan ahora las familias dentro de sus hogares y la creciente crisis de empleo ha generado, como ya señalamos, un incremento en los episodios de violencia doméstica.

Por otro lado, también se ha documentado que las mujeres estamos en mayor riesgo de sufrir consecuencias emocionales por el encierro. En México, entre 45 y 50 por ciento de las personas en aislamiento pueden presentar el "síndrome de cuarentena" que consiste en la manifestación de ansiedad, temor, irritabilidad, agresividad, apatía o incredulidad, estima Bernardo Solís, presidente de la Asociación Psiquiátrica Mexicana en una conferencia en línea que impartió a principios de abril ${ }^{12}$.

\footnotetext{
${ }^{12} \mathrm{https}: / / \mathrm{www} . e l s o l d e m e x i c o . c o m . m x / d o b l e-$ via/salud/ultimas-noticias-coronavirus-covid-19ansiedad-enfermedades-mentales-sintomasconsecuencias-confinamiento-pandemia-5188112.html
} 
México padece un grave problema de salud mental desde antes de que el coronavirus confinara a casi todos en sus casas. Los nuevos casos diagnosticados de depresión en hombres pasaron de 15 mil 849 en 2014 a 32 mil 649 el año pasado; y en mujeres, de 53 mil 745 a 93 mil 95 en el mismo lapso, revelan datos de los boletines epidemiológicos de la Secretaría de Salud federal $^{13}$.

Según el Consejo Ciudadano de la propia Secretaría de Salud, los sentimientos que más expresan los mexicanos durante la actual contingencia a partir del cuestionario para la detección de riesgos a las salud mental son ${ }^{14}$ :

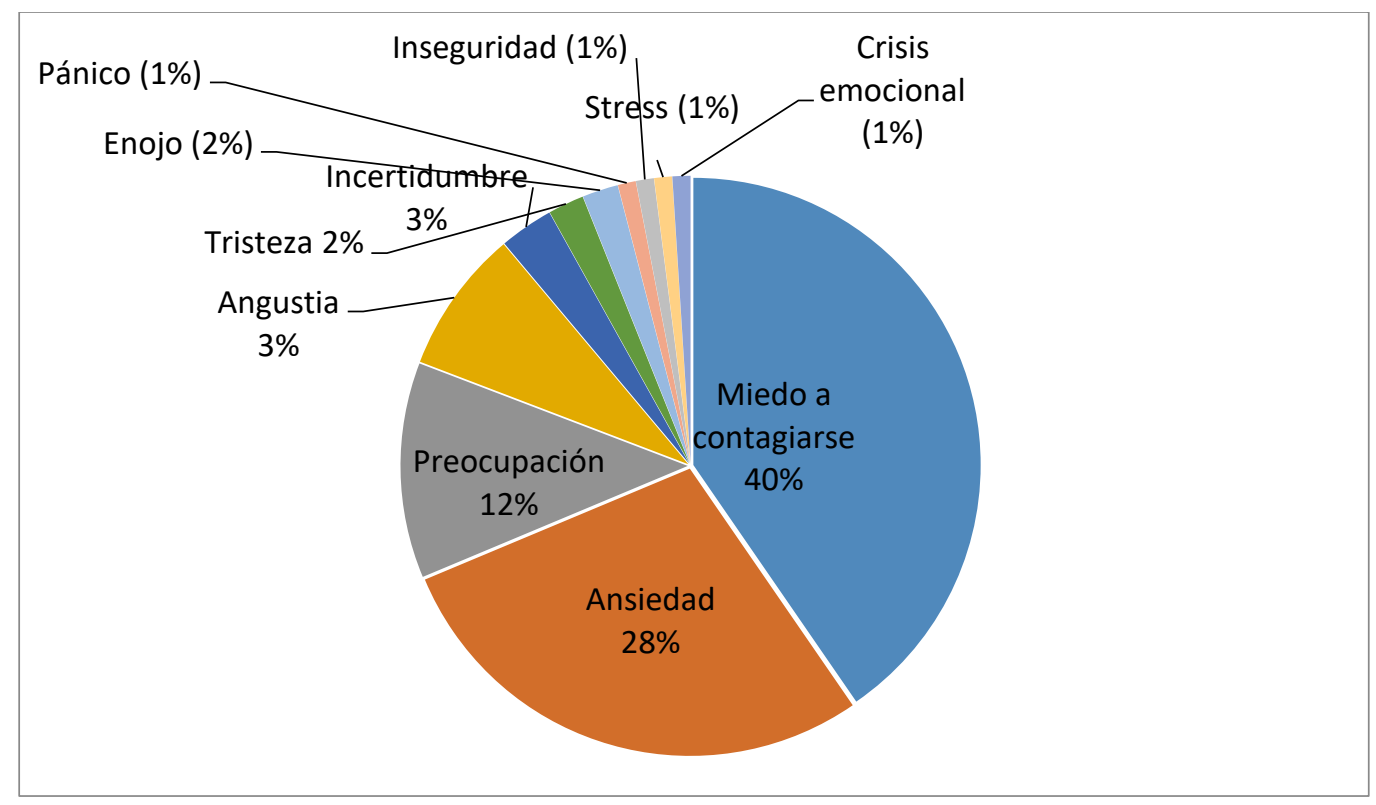

\section{Sobre el desafío educativo}

En cuanto a la deserción escolar encontramos cifras reveladoras: el Programa de las Naciones Unidas para el Desarrollo (PNUD) estima que para el ciclo escolar 2020-2021, alrededor de 1.4

\footnotetext{
${ }^{13} \mathrm{https} / / / \mathrm{www}$. elsoldemexico.com.mx/doblevia/salud/ultimas-noticias-coronavirus-covid-19ansiedad-enfermedades-mentales-sintomasconsecuencias-confinamiento-pandemia-5188112.html
}

millones de estudiantes en México no volverán a clases. Se estima que desertarán alrededor de 431,567 alumnos: 800,000 de secundaria; 593,000 universitarios y 38,567 de posgrado $^{15}$.

Encontramos una relación entre los niveles de abandono y el limitado acceso a los recursos digitales, sumado a la falta de

\footnotetext{
${ }^{14}$ https://misalud.unam.mx/covid19/

15 https://www.forbes.com.mx/la-pandemia-exhibedesigualdad-tecnologica-en-la-educacion-superiorpublica/
} 
apoyos para los profesores, pues a pesar de que un gran porcentaje de docentes se encuentra implementando educación por medios electrónicos, su dedicación no coincide con el apoyo recibido por parte de las instituciones educativas tanto en el sector público como privado, pues el $57.5 \%$ de los profesores declaró no tener ningún tipo de incentivo para llevar a cabo sus funciones durante la primera fase de la pandemia, lo cual puede repercutir también en la calidad de la educación pues muchos alumnos no han logrado adaptarse a estas nuevas modalidades.

En el caso de acceso a internet el INEGI advierte que únicamente $47 \%$ de los hogares tienen la posibilidad de hacer uso fijo $^{16}$, lo cual sumado a la dificultad que han tenido alumnos y docentes para retomar el ritmo escolar a través de estas nuevas tecnologías nos lleva a reflexionar sobre el gran desafío que tiene el país para alcanzar una educación de calidad y accesible para todos.

\section{Porcentaje de profesores que declaran no haber tenido apoyo para realizar labor docente vía remota}

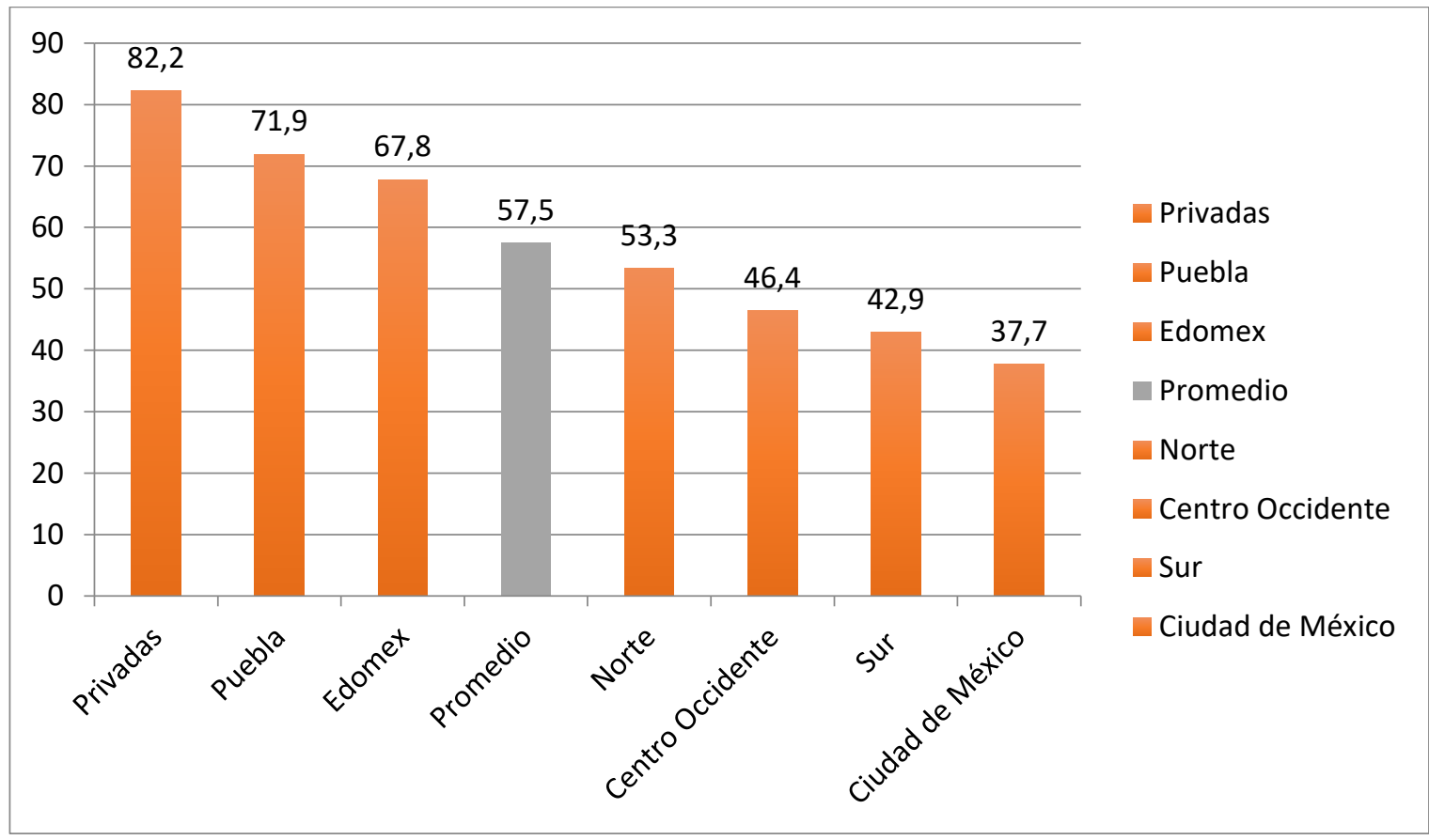

Elaboración propia a partir de datos de Nexos (https://educacion.nexos.com.mx/?p=2286)

\footnotetext{
$16 \mathrm{https} / / /$ www.milenio.com/politica/por-coronaviruspiden-evitar-abandono-escolar
} 


\section{Grado de dificultad para adaptarse a la educación a distancia}

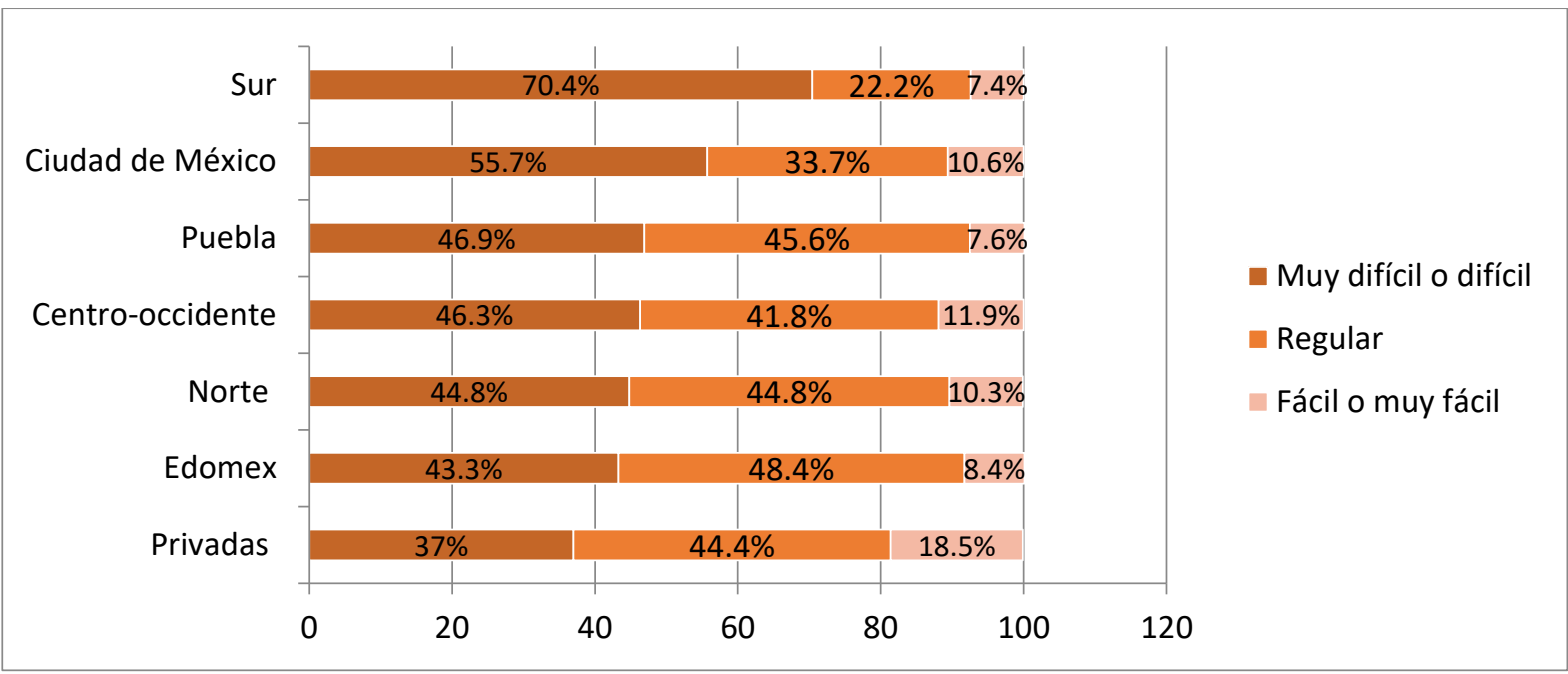

Elaboración propia a partir de datos de Nexos (https://educacion.nexos.com.mx/?p=2286)

\section{Percepción de apoyo por parte de alguna autoridad (pública o privada) para realizar labor docente durante la contingencia}

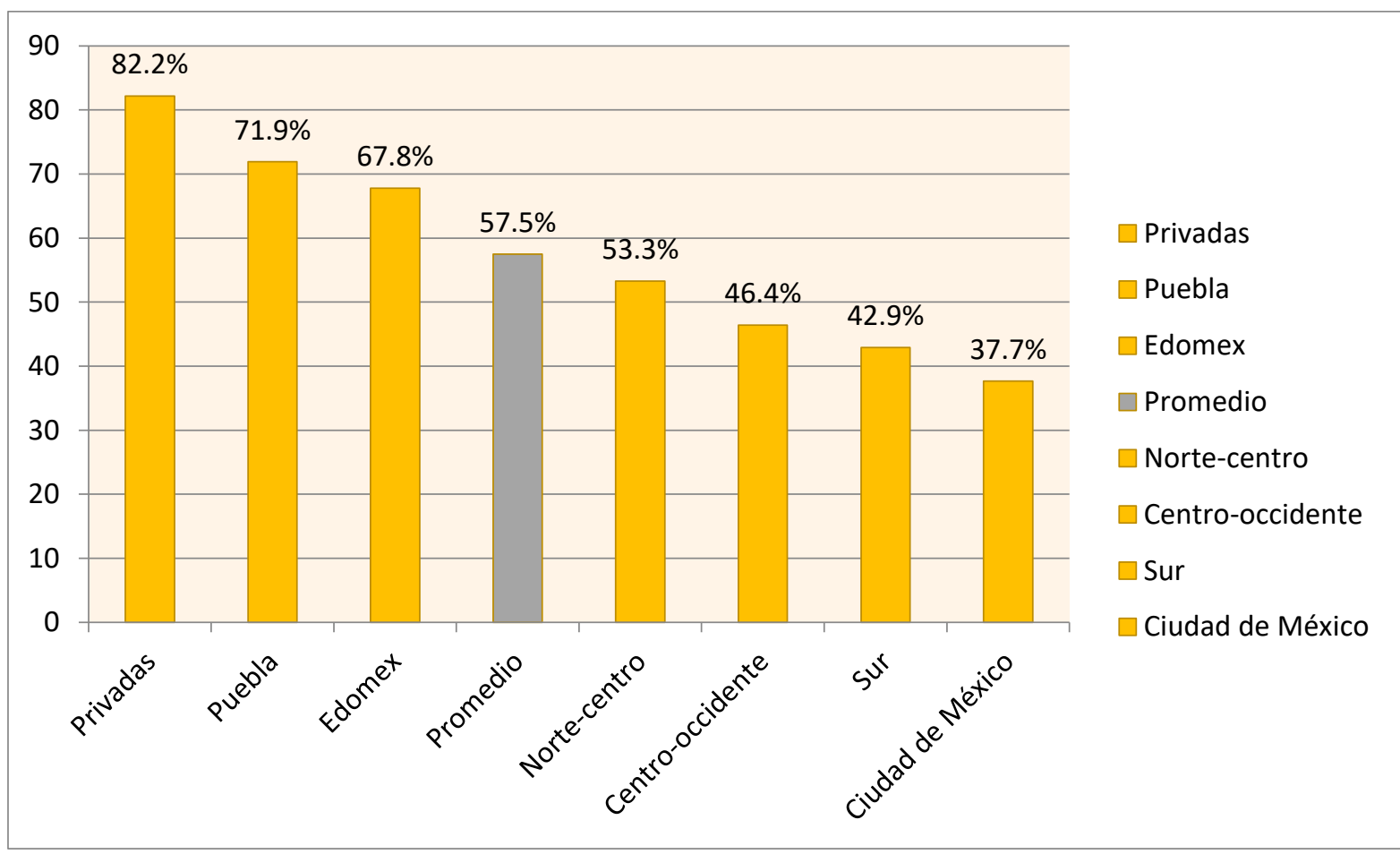

Elaboración propia a partir de datos de Nexos (https://educacion.nexos.com.mx/?p=2286)

Así como en otros países, en México se ha implementado el programa "Aprende en casa" que es una herramienta que permite que los alumnos continúen su educación desde su hogar a través de la transmisión de los contenidos de cada una de las materias dependiendo del grado escolar. La Secretaría de Educación Pública junto con 
el Sistema Público de Radiodifusión del Estado (SPR) y la Dirección General de Televisión Educativa lanzaron contenidos de educación preescolar, primaria, secundaria y bachillerato en la señal abierta, sin embargo tanto padres como alumnos y profesores no parecen estar muy satisfechos, pues la coordinación ha resultado insuficiente, sumado a que muchas veces los padres no tienen muy claro cómo implementar estos métodos, y no existen mecanismos de regulación para este novedoso método de aprendizaje. ${ }^{17}$

Debido a todas estas dificultades, algunos sectores han calificado la estrategia como un fracaso, pues no asegura el acceso a la educación debido no sólo a la falta de cobertura de internet en el país, sino que tampoco cuenta con acompañamiento eficaz por parte de docentes, lo cual repercute en procesos deficientes se enseñanza-aprendizaje, confiriendo más responsabilidades al estudiante, comprometiendo el tiempo de los padres y la interacción entre niños. Esto interfiriere en el intercambio de formas distintas de pensar, obstaculizando la construcción de una reflexión crítica. ${ }^{18}$

La pandemia ha cambiado la manera en que más de 25 millones de estudiantes

17 https://heraldodemexico.com.mx/nacional/2020/4/11/es cuela-en-casa-una-alternativa-para-continuar-con-elaprendizaje-167153.html reciben conocimiento y hasta ahora no tenemos certeza de cuando se terminará este gran reto que sin duda requiere de un esfuerzo conjunto entre todas las partes involucradas. Como señalamos, muchos docentes se sienten poco apoyados $y$ frustrados pues no cuentan con herrmientas suficientes apra interacatuar de manera remotea con sus estudiantes. Por otro lado, los padres señalan que emplean mucho de su tiempo en asegunrar la atención de sus hijos a las actividades escolares y no pueden apoyar de manera sustantiva la calidad de los contenidos que se espera que los niños aprendan. Por otra parte, los estudiantes señalan que tienen pocas oportunidades para preguntar cuando algo no les queda claro y que las tareas que tenen que enviar no equivalen a las actividades que antes realizaban en el salón de clase.

\section{Algunas historias ...}

El trabajo de campo remoto es un desafío en esta coyuntura. Sin embargo, logramos realizar entrevistas a familiares de personas que fallecieron por Covid-19 y que provienen de comunidades indígenas. Antes de recuperar fragmentos de estas entrevistas, nos parece importante señalar

18 https://aristeguinoticias.com/2104/mexico/programaaprende-en-casa-implementado-por-la-sep-un-fracasoseccion-22-de-la-cnte/ 
que muchas regiones indígenas en México, en particular en Oaxaca, han desarrollado estrategias para evitar los contagios por esta enfermedad. Muchas de estas medidas están motivadas por la carencia de servicios médicos en las regiones poco comunicadas o alejadas de centros urbanos y por la posibilidad que tienen los gobiernos llamados tradicionales de ejercer mayor control sobre la población local ${ }^{19}$.

\section{La familia de Flor}

Los hombres de la familia de Flor trabajan como soldados o policías. Es ya una tradición y las redes familiares les han permitido ascender, un poco, en este perfil de empleos a pesar de ser hablantes de Triqui una de las 68 lenguas mexicanas ${ }^{20}$. Casi toda la familia vive en la Ciudad de México, pero la primera generación aún radica en el municipio de Putla y con los dos ancianos vive una nieta, quien se hace cargo de sus cuidados. El resto de los integrantes de la familia envían dinero a Oaxaca para apoyar a los padres y para invertir en la producción agrícola local. El hermano mayor de Flor enfermó de Covid-19 los primeros días de abril del 2020. Probablemente se contagió en alguna de las

\footnotetext{
${ }^{19}$ Sobre este tema se ha discutido en trabajos previos sobre comunidades indígenas en contextos de migración y sobre las características de los gobiernos llamados de usos y costumbres (cfr. Martínez Casas y de la Peña 2004; Curiel 2017)

${ }^{20}$ Sobre el número de lenguas en México existe un largo debato. En 2008 el INAL publicó un catálogo de lenguas
}

instalaciones que le tocó visitar en esos días surtiendo material y equipo médico. Tenía 58 años y quería jubilarse, pero interrumpió los trámites y se ofreció como chofer para apoyar durante la contingencia sanitaria. La familia no reporta que sufriera alguna enfermedad previa. Llegó al hospital después de regresar a su casa con síntomas se insuficiencia respiratoria, pero tardó varios días en buscar ayuda médica. Dos días después de haber ingresado al hospital falleció. La familia no pudo realizar ningún ritual funerario y se llenó de tristeza. $\mathrm{Su}$ madre, en su comunidad, se sentía muy afligida y a finales del mes de julio se fracturó un brazo. Flor y su familia no tienen permitido regresar al pueblo y la nieta que se encarga de la abuela ha buscado apoyo, pero teme salir de la comunidad y que no le permitan regresar. Flor no le dicho a las personas con las que trabaja como empleada doméstica que falleció su hermano mayor de Covid, pues teme perder el empleo y ahora además de trabajar, se hace cargo de un nieto de tres años. Uno de sus hijos se divorció durante la pandemia. Su esposa le pidió el divorcio y uno de los argumentos que esgrimió es que la familia era contagiosa. Si la tragedia del

en donde recupera una manera diferente de clasificar la diversidad lingüística nacional. Allí se reporta la existencia de 11 familias lingüísticas con 68 agrupaciones y 364 variantes lingüísticas. No entraremos en ese debate que no ha sido resuelto desde hace muchas décadas. 
fallecimiento de su hermano mayor no fuera suficiente, a finales de septiembre otro de sus hermanos que trabajaba como policía también enfermó. No quiso ir al hospital y falleció en su casa. La necropsia que le realizaron indicó que no murió de Covid, sino de una infección que podría haber sido tratada de haber acudido al médico de manera oportuna.

\section{La familia de Lola}

El abuelo de Lola era comerciante a pesar de tener ya 82 años. Era una de las pocas personas a las que se les permitía entrar y salir de su comunidad en la Sierra Juárez de Oaxaca para surtir de productos su tienda. Sus dos hijos mayores se turnaban para traerlo y llevarlo entre la capital oaxaqueña y su comunidad. En junio del 2020 empezó con tos y algo de fiebre y las autoridades locales le pidieron a la familia que lo sacara de la comunidad para que recibiera atención médica. Lo hospedó Lola en la ciudad de Oaxaca y al cabo de una semana toda la familia tenía los mismos síntomas que el abuelo quien, finalmente, requirió hospitalización. Después de 10 días con un ventilador el señor falleció. Tampoco pudieron realizar rituales funerarios y la familia en la comunidad se enojó con Lola y sus hermanos por haberlo llevado al hospital. Al momento de las entrevistas, dos hermanos de Lola seguían enfermos y las cenizas del anciano esperaban en una urna poder regresar a su pueblo. La tristeza y el enojo se mezclaron en la familia y se culpan por no haberle permitido morir en su casa. En la comunidad no les permiten regresar.

\section{La familia de Susana}

Susana y su familia son la segunda generación radicados en la Ciudad de México. Sus padres llegaron desde Huajuapan, Oaxaca en la década de 1970 y se instalaron en un barrio de la Alcaldía de Coyoacán con muchas personas de su región y se siguen comunicando en Mixteco. Hace 20 años una de las tías de Susana migró también a la Ciudad de México y se instaló con su familia y encontró empleo como apoyo de limpieza en unas oficinas gubernamentales. En julio del 2020 se rompió la cadera y requirió una cirugía que se llevó a cabo en un hospital público. Estaba aterradame narró Susana- porque temía enfermar en el hospital y sus temores se hicieron realidad. La dieron del alta y una semana después tuvo que ser ingresada de nuevo con síntomas de Covid-19. Sobrevivió cinco días, pero desde que la internaron ella se despidió de su familia. Pensaba que no volvería a verlos y solo habló de nuevo con ellos por video llamada en una ocasión. La señora tenía 57 años y la familia reporta que no tenía otra enfermedad y que se había fracturado al 
caerse de una escalera. Estaba asistiendo a laborar a la oficina porque se consideró que no pertenecía a algún grupo de riesgo, mientras que la mayoría de los empleados públicos en México fueron enviados a sus casas durante la contingencia sanitaria. Susana recibió las cenizas de su tía en una cajita de cartón y afirma que no está segura que se trate de sus restos porque no le entregaron la ropa con la que ingresó al hospital y no tiene manera de garantizar que se trate de ella. En la casa familiar llevaron a cabo rosarios y esperan que les den fecha para una misa en la parroquia. Susana es auxiliar de enfermería y no sabe si ella contagió a su tía o se enfermó tras ser operada en el hospital, pues dio positivo en una prueba unos días después de que su tía enfermara.

Las tres historias reflejan lo que se ha documentado en mucha de la literatura antropológica sobre la relación saludenfermedad en comunidades indígenas en México $^{21}$. Los padecimientos de salud afectan a toda la familia y la afectación se extiende a todas las esferas de su vida. También se ha documentado que la pandemia por Covid-19 afecta más a las personas que provienen de pueblos indígenas y que tiene difícil acceso al sistema de salud. Los tres casos que se reseñan muestran que, con frecuencia, los

\footnotetext{
${ }^{21}$ Para una discusión sobre estos temas ver Martínez Casas 2007.
}

indígenas en México se emplean en tareas que los ponen en riesgo de contagio y ninguno de ellos sufría alguna enfermedad agravante. Se trata de dos adultos y un anciano, pero su principal factor de riesgo era su empleo. El otro factor común en las tres historias es el estigma que ha afectado no solo a quienes se contagiaron $\mathrm{o}$ fallecieron sino a toda su familia y que ha formado parte de la construcción social de esta crisis de salud en México y el mundo.

\section{Reflexiones finales}

En el presente texto buscamos mostrar algunos de los efectos de la pandemia por Covid-19 en México a partir del análisis de algunas de las comunicaciones oficiales sobre el tema. Encontramos que la estigmatización que existe alrededor de los contagios y fallecimientos proviene, en parte, de la retórica utilizada por los funcionarios gubernamentales que culpan a la propia población por la crisis sanitaria. En una primera etapa el énfasis estaba en el confinamiento. Quienes salían de sus casas eran responsables de la trasmisión acelerada de la enfermedad, pero quienes se agravaban o morían era por su descuido. Las personas con sobrepeso, hipertensión o diabetes eran responsables de sus propias muertes. 
Cuando se publicaron las cifras sobre contagios y fallecimientos en el personal de salud la retórica cambió. El manejo de la pandemia ha sido exitoso porque se ha impedido la saturación hospitalaria y se hicieron esfuerzos para la compra de equipos en un momento en el que el mundo entero los requería. En las conferencias vespertinas se empezó a hablar de estrategias de prevención ante rebrotes y la inminente llegada de la influenza estacional y se dejaron de lado los análisis sobre comorbilidades. Pero para ese momento México ocupaba ya el cuarto lugar a nivel mundial en número de muertes y una tasa de mortalidad superior al $10 \%$ de los contagios confirmados. Cuando en Europa están ya padeciendo una segunda oleada de contagios, en México el panorama se ha mantenido estable, pero los contagios y fallecimientos no han disminuido de manera significativa.

Por otro lado, las consecuencias en la economía y la educación afectaron de manera más grave a las mujeres y el impacto en el desempleo y la capacidad de gasto de las familias es todavía incalculable. Una variable que no mencionamos con anterioridad es que una de las pocas fuentes de recursos económicos que nunca disminuyó fue el ingreso de remesas desde Estados Unidos, de tal suerte que en septiembre de 2020 era $23 \%$ más altas que en el mismo mes de 2019. El monto promedio no es superior, pero sí el número de mexicanos radicados en Estados Unidos que están enviando sus ahorros para apoyar a sus familias en México. También es importante mencionar que el efecto en la atención a otros temas de salud afectó de manera más aguda a las mujeres. Se incrementó $46 \%$ la tasa de muerte materna, por primera vez en la historia de México el número de cesáreas superó la de partos por vía vaginal, disminuyo más de $30 \%$ la contabilización de diagnósticos de cáncer mama y más de la mitad de las mujeres que ya recibían tratamiento por este padecimiento - una de las primeras causas de muerte femenina en México- lo interrumpió.

También quisimos ilustrar con algunas historias cercanas a nosotros el drama que ha representado para decenas de miles de familias el tener que lidiar con el estigma de la enfermedad, la angustia de la agonía en soledad y el drama de muertes que cargan con una serie de consecuencias para comunidades enteras. Si bien son breves descripciones etnográficas basadas en entrevistas realizadas de manera remota, su incorporación en el texto busca darle una cara humana a las cifras que se presentan en las diferentes secciones del trabajo. 
Hacia mediados de octubre las contradicciones entre el presidente de la república y el subsecretario de Salud se volvieron tan evidentes que el Dr. López Gatell tuvo que aclarar que se trataba de un problema de perspectiva y que el presidente tiene la obligación de mirar el conjunto de los grandes temas nacionales, mientras que a él le corresponde centrarse en la contingencia sanitaria. El hecho es que los funcionarios de la Secretaría de Salud y los gobiernos locales afirman que México vive ya un rebrote y siguen alentando a que las personas retomen el encierro, mientras que el titular del poder ejecutivo insiste en que la pandemia está bajo control.

Todavía es muy pronto para hacer un recuento de todas las afectaciones en la vida del país y de los mexicanos en esta difícil coyuntura que no solo se puede clasificar como una crisis de salud. Es y será por años una crisis social -que ha afectado gravemente a las mujeres y a la población en edad escolar- y una crisis económica. La realidad es que la molestia de las mujeres y los problemas económicos se habían gestado antes de la pandemia y se han agravado durante estos más de 250 días de contingencia y ni la retórica gubernamental que minimiza la gravedad de la crisis ni el panorama mundial ayudan a vislumbrar una salida al corto plazo.

\section{Referências bibliográficas}

Curiel, Charlynne (2017) De la comunidad corporada a la comundad frgamentada: Proyectos comunitarios en disputa. Editorial Juan Pablos, México.

Esteban, Mari Luz, (2017), "Los cuidados, un concepto central en la teoría feminista: aportaciones, riesgos y diálogos con la antropología", Quaderns-e de l'Institut Català d'Antropologia, 22(2), Barcelona: ICA, pp. 33-48.

Martínez Casas, Regina (2007) Vivir invisibles: la resignificación cultural entre los otomíes urbanos en Guadalajrar. CIESAS; México.

Martínez Casas, Regina y Guillermo de la Peña (2004) Migrantes y comunidades morales. Resignificaión, etnicidad y redes sociales. En Revista de Antropología Social. Universidad Complutense de Madrid. Vol 13, pp: 217-251.

Rodríguez, Corina. (2015), "Economía feminista y economía del cuidado. Aportes conceptuales para el estudio de la desigualdad", Argentina: Nueva Sociedad, pp. 30-44.

El Universal [El Universal]. (2020, abril 27), La mañanera de AMLO [Archivo de video]. Recuperado de https://www.youtube.com/watch?v=Ub2uE XFv1lg

Redacción (29 de abril de 2020). Artistegui noticias, México. Recuperado de https://aristeguinoticias.com/2904/mexico/ amlo-dijo-que-se-esta-aplanando-la-curvapero-en-la-fase-3-los-contagios-sonexponenciales-video/

Presidencia de la República (4 de mayo de 2020). Conferencia de prensa. Informe diario sobre coronavirus COVID-19 en México, México. Recuperado de https://www.gob.mx/presidencia/es/articul 
os/version-estenografica-conferencia-deprensa-informe-diario-sobre-coronaviruscovid-19-en-mexico-241579?idiom=es

Redacción (9 de junio de 2020). México da ejemplo al mundo en control de epidemia de COVID 19: presidente; manejo ha sido eficaz y con apoyo del pueblo, afirma, México. Recuperado de: https://lopezobrador.org.mx/2020/06/09/m exico-da-ejemplo-al-mundo-en-control-deepidemia-de-covid-19-presidente-manejoha-sido-eficaz-y-con-apoyo-del-puebloafirma/

Gutiérrez, Noemi (7 de julio de 2020). AMLO: se logró aplanar la curva y no se desbordó el sistema de salud, lo que ha significado salvar vidas, "no ha sido en vano el sacrificio de la gente", México. Recuperado de https://www.reporteindigo.com/reporte/am lo-se-logro-aplanar-la-curva-de-contagiosno-ha-sido-en-vano-el-sacrificio-de-lagente/

Urrutia, Alonso; Muñoz, Alma (15 de julio de 2020). Estrategia del gobierno, "clave para aplanar la curva": AMLO, México. Recuperado de:

https://www.jornada.com.mx/ultimas/politi ca/2020/07/15/estrategia-del-gobiernoclave-para-aplanar-la-curva-amlo1284.html

Redacción (2 de septiembre de 2020). México, primer lugar mundial en personal de salud fallecido por COVID-19:

Amnistía Internacional, México.

Recuperado de:

https://www.elfinanciero.com.mx/salud/me xico-primer-lugar-mundial-en-personal-desalud-fallecido-por-covid-19-amnistiainternacional

Forbes Staff (7 de agosto de 2020). AMLO descarta cambiar estrategia contra COVID19: 'se aplanó la curva', insiste, México. Recuperado de: https://www.forbes.com.mx/politica-amloestrategia-covid-19-aplano-curva/

Reynoso, Alejandra (27 de julio de 2020). Alertan que crisis por coronavirus expone a más violencia a mujeres, México.

Recuperado de https://www.milenio.com/politica/comunid $\mathrm{ad} /$ coronavirus-alertan-crisis-exponeviolencia-mujeres

Redacción (9 de julio de 2020) Se perdieron en México más de 12 millones de empleos en dos meses, México. Recuperado de https://www.elfinanciero.com.mx/economi a/se-perdieron-en-mexico-mas-de-12millones-de-empleos-en-dos-meses-segundatos-de-banxico

Hernández, Saúl (5 de mayo de 2020) Enfermedades mentales, el lado oscuro del Covid-19, México. Recuperado de https://www.elsoldemexico.com.mx/doble$\mathrm{via} / \mathrm{salud} / \mathrm{ultimas}$-noticias-coronaviruscovid-19-ansiedad-enfermedadesmentales-sintomas-consecuenciasconfinamiento-pandemia-5188112.html

https://misalud.unam.mx/covid19/

Ordaz, Arturo (15 de mayo de 2020) La pandemia exhibe desigualdad tecnológica en la educación superior pública, México. Recuperado de https://www.forbes.com.mx/la-pandemiaexhibe-desigualdad-tecnologica-en-laeducacion-superior-publica/

Wong, Ana (15 de abril de 2020) Llama Mejoredu a evitar abandono escolar como consecuencia de la pandemia, México. Recuperado de https://www.milenio.com/politica/porcoronavirus-piden-evitar-abandono-escolar

Heraldo de México (11 de abril de 2020)

Escuela en casa: una alternativa para continuar con el aprendizaje, México. Recuperado de: https://heraldodemexico.com.mx/nacional/ 
2020/4/11/escuela-en-casa-una-alternativapara-continuar-con-el-aprendizaje-

167153.html

Redacción (21 de abril de 2020) Programa "Aprende en casa" implementado por la SEP, "un fracaso: Sección 22 de la CNTE, México. Recuperado de:

https://aristeguinoticias.com/2104/mexico/ programa-aprende-en-casa-implementadopor-la-sep-un-fracaso-seccion-22-de-lacnte/ 